\title{
COLLECTION OF THE PLANT SYMPHYOTRICHUM NEES GENUS IN VYTAUTAS MAGNUS UNIVERSITY BOTANICAL GARDEN
}

\author{
Indrė LUKŠYTĖ, Institute of Biology and Plant Biotechnology, Agriculture Academy, Vytautas Magnus University. K. Donelaičio \\ str. 58, LT-44248, Kaunas, indre.luksyte@ vdu.lt \\ Sonata KAZLAUSKAITÉ, Institute of Biology and Plant Biotechnology, Agriculture Academy, Vytautas Magnus University. K. \\ Donelaičio str. 58, LT-44248, Kaunas, sonata.kazlauskaite@ vdu.lt (corresponding author) \\ Arūnas BALSEVIČIUS, Vytautas Magnus University. K. Donelaičio str. 58, LT-44248, Kaunas, Lithuania, \\ arunas.balsevicius@vdu.lt \\ Ričardas NARIJAUSKAS, Vytautas Magnus University. K. Donelaičio str. 58, LT-44248, Kaunas, Lithuania, \\ ricardas.narijauskas@vdu.lt
}

The collection of 82 collection numbers of the plant Symphyotrichum Nees genus has been accumulated in the period of 1923 - 2018 at Vytautas Magnus University Botanical Garden. The collection consists of Symphyotrichum cordifolium, S. dumosum, S. ericoides, S. laeve, S. lanceolatum, S. lateriflorum, S. novae-angliae, S. novi-belgii, S. oblongifolium, S. pilosum, S. $\times$ salignum, S. tradescantii, S. turbinellum, $S$. urophyllum, and their infraspecific taxa and cultones. The major part of the collection consists of $S$. novi-belgii (32 collection numbers) and $S$. novae-angliae (19 collection numbers) infraspecific taxa and cultones. 57 collection numbers were acquired by sproutings from other botanical gardens, private collections, nurseries, the origin of 21 collection numbers is unknown, 4 collection numbers were acquired by seed exchange with other botanical gardens. Phenological observations and biometric measurements of plants were performed according to methodological manual "Methodology of phenological observations, biometric measurements and assortment formation of ornamental herbaceous plants" prepared by J. Vaidelys in 2005. When assessing the phytopathological status of plants, powdery mildew (Erysiphe cichoracearum) was determined as the main disease affecting the plants. S. dumosum and S. novibelgii interspecific taxa and cultones were the most susceptible to the disease. Plants grown in the same location for more than 3 years were more susceptible to the disease. The aim of the study was to review the Symphyotrichum collection and to evaluate the susceptibility of different groups of cultivars to powdery mildew.

Keywords: Symphyotrichum, introduction, collection, phytopathological state, powdery mildew.

\section{INTRODUCTION}

Representatives of the Symphyotrichum Nees genus are annual and perennial herbaceous plants belonging to the family of Asteraceae Bercht. \& J. Presl. According to the Plant List Taxonomic Standardization System (Cayuela, Oksanen, 2016), there are 108 approved species in the genus, which are most widespread in North America, with several species naturally growing in West India, Central and South America, Eurasia. Representatives of Symphyotrichum were assigned to the genus Aster L. until 1833. However, a botanist Christian Gottfried Daniel Nees von Esenbeck distinguished the genus Symphyotrichum in 1833, when he had observed that some Aster species were morphologically distinct from other species. Guy Nesom updated the taxonomic classification of Symphyotrichum in 1994 (Nesom, 1994).

All representatives of Symphyotrichum are herbaceous plants that bloom in the second half of summer until late autumn. The breeders created more than 300 cultivars with flower colours ranging from white to dark purple. There are even hybrids with yellow flowers, therefore, these are rare cultivars. Symphyotrichum plants are appreciated for their easy maintenance, decorativeness, and resistance to adverse weather conditions. Especially since they are one of the few plants that flower during the autumn season. Moreover, Symphyotrichum plants are easy to proliferate and therefore are appreciated by flower growers (Hertle et al., 1996). Although Symphyotrichum plants are relatively resistant to adverse environmental conditions, powdery mildew (caused by Erysiphe cichoracearum) is recognized as one of the main problems causing loss of plant decorativeness (Pirone, 1978; Kazlauskaite, 2012). For this reason, resistance to powdery mildew is one of the most important criteria when developing new Symphyotrichum cultivars.

\section{METHODS}

The classification and estimation of Symphyotrichum grown in Vytautas Magnus University Botanical Garden was performed according to the Plant List Taxonomic Standardization System (Cayuela, Oksanen, 2016). Plant observations were carried out in accordance with "Methodology of phenological observations, biometric measurements and assortment formation of ornamental herbaceous plants" prepared by J. Vaidelys in 2005. Phytopathological state of plants was

Copyright $(0) 2019$ The Authors. Published by Vytautas Magnus University. This is an open-access article distributed under the terms of the Creative Commons Attribution License (CC BY 4.0), which permits unrestricted use, distribution, and reproduction in any medium, provided the original author and source are credited. 
monitored by regular inspection of all plants in the collection. Plant diseases were identified according to manuals "Plant Protection in Landscape" (Kazlauskaite, 2012) and "Diseases and Pests of Ornamental Plants" (Pirone, 1978).

\section{RESULTS}

The collection of Symphyotrichum spp. was started in 1923 when Kaunas Botanical Garden was established (now Vytautas Magnus University Botanical Garden). The major part of the collection consists of S. novi-belgii (32 collection numbers) and $S$. novae-angliae (19 collection numbers) (Table 1).

Table 1. Collection of Symphyotrichum spp. at Vytautas Magnus University Botanical Garden in 2019.

\begin{tabular}{|c|c|c|}
\hline Species & Infraspecific taxon & Cultone \\
\hline S. cordifolium & & 'Little Carlow' \\
\hline S. dumosum & & $\begin{array}{l}\text { 'Amoranth', 'Andry', 'Damelight', 'Diana', 'Herbstarus vom Besserhof', 'Herbstgrüb } \\
\text { vom Bresserhof', 'Herbstrot', 'Ronald' }\end{array}$ \\
\hline S. ericoides & & 'First Snow', 'Golden Spray‘, 'Weisser Zwerg' \\
\hline S. laeve & & 'Blue Bird' \\
\hline S. lanceolatum & var. lanceolatum & \\
\hline S. lateriflorum & & 'Lady in Black', 'Prince' \\
\hline S. novae-angliae & & $\begin{array}{l}\text { 'Alba', 'Andenken an Alma Pötschke', 'Bars Pink', 'Cardinal Tarcisio Bertone', } \\
\text { 'Dapper Tapper', 'Erfurt Blut', 'Harington Pink', 'Herbstschnee', 'Kylie', 'Lye End } \\
\text { Beauty', 'Primrose Upward', 'Purple Dome', 'Rosa Sieger', 'Röter Turm', } \\
\text { 'Ruderburg', 'Violetta', 'W. Bowman' }\end{array}$ \\
\hline S. novi-belgii & var. novi-belgii & $\begin{array}{l}\text { 'Apollo', Bahamas, Barbados, 'Belosnezka', 'Blauer Vorläufer', 'Crimson Brocade', } \\
\text { 'Fuldatal', 'Jenny', 'Kassel', 'Kristina', 'Lady in Blue', 'Lilac Time', 'Magic Blue', } \\
\text { 'Marie Ballard', 'Melbourne', 'Patricia Ballard', 'Pink Lace', 'Professor Anton } \\
\text { Kippenberg', 'Rose Bonnet', 'Rosenwichtel', 'Royal Ruby', 'Rosalinde', } \\
\text { 'Rosenponpon', Samoa, 'Snowdrift', 'Snowsprite', 'Strawberries and Cream', } \\
\text { Tonga, 'White Ladies' }\end{array}$ \\
\hline \multicolumn{3}{|l|}{ S. oblongifolium } \\
\hline \multicolumn{3}{|l|}{ S. pilosum } \\
\hline \multicolumn{3}{|l|}{ S. $\times$ salignum } \\
\hline \multicolumn{3}{|l|}{ S. tradescantii } \\
\hline S. turbinellum & & 'JS Leaflet' \\
\hline S. urophyllum & & 'Ann Leys', 'Newstars Glory', 'Pink Star', 'Woods Purple' \\
\hline
\end{tabular}

Symphyotrichum spp. collection has been collected through seed exchange with other botanical gardens as well as living plants were purchased from private collections, nurseries, and botanical gardens in Lithuania, Latvia, Estonia, Great Britain, Germany, Ukraine, Croatia, Hungary. S. cordifolius was recognized as the oldest representative growing in the collection (Table 2). The plant has been purchased from the Botanical Garden of the Justus Liebig University Giessen due to the Seed Exchange Program in 1979.

Table 2. Introduction of Symphyotrichum spp. at Vytautas Magnus University Botanical Garden.

\begin{tabular}{|l|l|l|l|}
\hline \multicolumn{1}{|c|}{ Species } & \multicolumn{1}{|c|}{$\begin{array}{c}\text { Year of } \\
\text { acquisition }\end{array}$} & $\begin{array}{c}\text { Country of } \\
\text { acquisition }\end{array}$ & \multicolumn{1}{|c|}{ Authority from which the material was obtained } \\
\hline S. cordifolium & 1979 & Germany & Botanical Garden of the Justus Liebig University Giessen \\
\hline S. cordifolium 'Little Carlow' (cordifolium hybrid) & 2012 & Lithuania & Dariaus Gèlès Ltd, Kaunas \\
\hline S. dumosum 'Amoranth' & Unknown & Unknown & Unknown \\
\hline S. dumosum 'Andry' & Unknown & Unknown & Unknown \\
\hline S. dumosum 'Damelight' & Unknown & Unknown & Unknown \\
\hline S. dumosum 'Diana' & Unknown & Unknown & Unknown \\
\hline S. dumosum 'Herbstarus vom Besserhof' & 2014 & Latvia & National Botanic Garden of Latvia, Salaspils \\
\hline S. dumosum 'Herbstgrüb vom Bresserhof' & Unknown & Unknown & Unknown \\
\hline S. dumosum 'Herbstrot' & Unknown & Unknown & Unknown \\
\hline S. dumosum 'Ronald' & 2015 & Lithuania & Parko medelynas Ltd, Marijampole \\
\hline S. ericoides & Unknown & Unknown & Unknown \\
\hline S. ericoides 'First Snow' & 2015 & Lithuania & Vyšniauskai floriculture farm, Raseiniai \\
\hline S. ericoides 'Golden Spray' & 2014 & Lithuania & Vyšniauskai floriculture farm, Raseiniai \\
\hline
\end{tabular}




\begin{tabular}{|c|c|c|c|}
\hline Species & $\begin{array}{c}\text { Year of } \\
\text { acquisition }\end{array}$ & $\begin{array}{l}\text { Country of } \\
\text { acquisition }\end{array}$ & Authority from which the material was obtained \\
\hline S. ericoides 'Weisser Zwerg' & 2015 & Lithuania & Vyšniauskai floriculture farm, Raseiniai \\
\hline S. laeve 'Blue Bird' & 1983 & Ukraine & $\begin{array}{llll}\text { Dnipropetrovsk } & \text { University } & \text { Botanical } & \text { Garden, } \\
\text { Dnipropetrovsk } & & & \end{array}$ \\
\hline S. laeve 'Blue Bird' & 2000 & Croatia & Faculty of Science, University of Zagreb, Zagreb \\
\hline S. lanceolatum var. lanceolatum & 1989 & Estonia & University of Tartu Botanical Gardens, Tartu \\
\hline S. lateriflorum 'Lady in Black' & 2013 & Lithuania & Dariaus Gèlès Ltd, Kaunas \\
\hline S. lateriflorum 'Lady in Black' & 2015 & Lithuania & Parko medelynas Ltd, Marijampolè \\
\hline S. lateriflorum 'Prince' & 2015 & Lithuania & Parko medelynas Ltd, Marijampolè \\
\hline S. novae-angliae 'Alba' & 2013 & Unknown & Unknown \\
\hline S. novae-angliae 'Andenken an Alma Pötschke' & 2007 & Latvia & National Botanic Garden of Latvia, Salaspils \\
\hline S. novae-angliae 'Bars Pink' & 2013 & Unknown & Unknown \\
\hline S. novae-angliae 'Bars Pink' & Unknown & Unknown & Unknown \\
\hline S. novae-angliae 'Cardinal Tarcisio Bertone' & 2015 & Lithuania & Parko medelynas Ltd, Marijampolè \\
\hline S. novae-angliae 'Dapper Tapper' & 2014 & Lithuania & Vyšniauskai floriculture farm, Raseiniai \\
\hline S. novae-angliae 'Erfurt Blut' & Unknown & Unknown & Unknown \\
\hline S. novae-angliae 'Harington Pink' & Unknown & Unknown & Unknown \\
\hline S. novae-angliae 'Herbstschnee' & 2015 & Lithuania & Parko medelynas Ltd, Marijampolè \\
\hline S. novae-angliae 'Herbstschnee' & 2015 & Lithuania & Vyšniauskai floriculture farm, Raseiniai \\
\hline S. novae-angliae 'Kylie' & 2014 & Lithuania & Vyšniauskai floriculture farm, Raseiniai \\
\hline S. novae-angliae 'Lye End Beauty' & 2014 & Lithuania & Vyšniauskai floriculture farm, Raseiniai \\
\hline S. novae-angliae 'Primrose Upward' & 2014 & Lithuania & Vyšniauskai floriculture farm, Raseiniai \\
\hline S. novae-angliae 'Purple Dome' & 2014 & Great Britain & Market, Ely \\
\hline S. novae-angliae 'Rosa Sieger' & 2012 & Lithuania & Dariaus Gèlès Ltd, Kaunas \\
\hline S. novae-angliae 'Röter Turm' & 2013 & Latvia & Botanical Garden of the University of Latvia, Riga \\
\hline S. novae-angliae 'Ruderburg' & Unknown & Unknown & Unknown \\
\hline S. novae-angliae 'Violetta' & 2014 & Lithuania & Dariaus Gèlès Ltd, Kaunas \\
\hline S. novae-angliae 'W. Bowman' & 1981 & Unknown & Unknown \\
\hline S. novi-belgii var. novi-belgii & 1987 & Hungary & Unknown \\
\hline S. novi-belgii 'Apollo' & 2017. & Lithuania & Parko medelynas Ltd, Marijampolè \\
\hline S. novi-belgii Bahamas & 2012 & Lithuania & Dariaus Gèlès Ltd, Kaunas \\
\hline S. novi-belgii Barbados & 2017. & Lithuania & Parko medelynas Ltd, Marijampolė \\
\hline S. novi-belgii 'Belosnezka' & 2012 & Lithuania & Kęstutis Volkus private collection, Kèdainiai \\
\hline S. novi-belgii 'Blauer Vorläufer' & 2013 & Latvia & Botanical Garden of the University of Latvia, Riga \\
\hline S. novi-belgii 'Crimson Brocade' & 2015 & Lithuania & Vyšniauskai floriculture farm, Raseiniai \\
\hline S. novi-belgii 'Fuldatal' & 2014 & Latvia & National Botanic Garden of Latvia, Salaspils \\
\hline S. novi-belgii 'Jenny' & 2014 & Lithuania & Dariaus Gèlès Ltd, Kaunas \\
\hline S. novi-belgii 'Jenny' & 2003 & Lithuania & Monika Sadauskienė private collection, Kaunas \\
\hline S. novi-belgii 'Kassel' & 2015 & Lithuania & Dariaus Gèlès Ltd, Kaunas \\
\hline S. novi-belgii 'Kristina' & 1987 & Unknown & Unknown \\
\hline S. novi-belgii 'Lady in Blue' & 2014 & Estonia & Tallinn Botanical Garden, Tallinn \\
\hline S. novi-belgii 'Lilac Time' & Unknown & Unknown & Unknown \\
\hline S. novi-belgii 'Magic Blue' & 2013 & Lithuania & Augustė Mieldažienė private collection, Kazlų Rūda \\
\hline S. novi-belgii 'Marie Ballard' & 2011 & Lithuania & Danielè Šležienè private collection, Vilnius \\
\hline S. novi-belgii 'Melbourne' & 1987 & Hungary & Unknown \\
\hline S. novi-belgii 'Patricia Ballard' & 2015 & Lithuania & Vyšniauskai floriculture farm, Raseiniai \\
\hline S. novi-belgii 'Pink Lace' & 2013 & Latvia & Botanical Garden of the University of Latvia, Riga \\
\hline S. novi-belgii 'Professor Anton Kippenberg' & Unknown & Unknown & Unknown \\
\hline S. novi-belgii 'Rose Bonnet' & 2014 & Estonia & Tallinn Botanic Garden, Tallinn \\
\hline
\end{tabular}




\begin{tabular}{|c|c|c|c|}
\hline Species & $\begin{array}{c}\text { Year of } \\
\text { acquisition }\end{array}$ & $\begin{array}{l}\text { Country of } \\
\text { acquisition }\end{array}$ & Authority from which the material was obtained \\
\hline S. novi-belgii 'Rosenwichtel' & 2011 & Lithuania & Angelė Žiukienė private collection, Kaunas \\
\hline S. novi-belgii 'Royal Ruby' & 2014 & Lithuania & Dariaus Gèlès Ltd, Kaunas \\
\hline S. novi-belgii 'Rosalinde' & 1987 & Hungary & Unknown \\
\hline S. novi-belgii 'Rosenponpon' & 2015 & Lithuania & Vyšniauskai floriculture farm, Raseiniai \\
\hline S. novi-belgii Samoa & 2017. & Lithuania & Parko medelynas Ltd, Marijampole \\
\hline S. novi-belgii 'Snowdrift' & Unknown & Unknown & Unknown \\
\hline S. novi-belgii 'Snowsprite' & 2014 & Great Britain & Market, Ely \\
\hline S. novi-belgii 'Strawberries and Cream' & 2015 & Lithuania & Vyšniauskai floriculture farm, Raseiniai \\
\hline S. novi-belgii Tonga & 2012 & Lithuania & Dariaus Gèlès Ltd, Kaunas \\
\hline S. novi-belgii Tonga & 2017. & Lithuania & Parko medelynas Ltd, Marijampolè \\
\hline S. novi-belgii 'White Ladies' & 2012 & Lithuania & Dariaus Gèlès Ltd, Kaunas \\
\hline S. oblongifolium & 2014 & Latvia & National Botanic Garden of Latvia, Salaspils \\
\hline S. pilosum & 2014 & Latvia & National Botanic Garden of Latvia, Salaspils \\
\hline S. $\times$ salignum & 2015 & Lithuania & Vyšniauskai floriculture farm, Raseiniai \\
\hline S. tradescantii & 1983 & Unknown & Unknown \\
\hline S. turbinellum 'JS Leaflet' & 2015 & Lithuania & Vyšniauskai floriculture farm, Raseiniai \\
\hline S. urophyllum & 2014 & Latvia & National Botanic Garden of Latvia, Salaspils \\
\hline S. 'Ann Leys' & 2015 & Lithuania & Parko medelynas Ltd, Marijampolè \\
\hline$S$. 'Newstars Glory' (Newstars Series) & 2014 & Lithuania & Vyšniauskai floriculture farm, Raseiniai \\
\hline S. 'Pink Star' & 2015 & Lithuania & Vyšniauskai floriculture farm, Raseiniai \\
\hline S. 'Pink Star' & 2015 & Lithuania & Parko medelynas Ltd, Marijampolè \\
\hline S. 'Woods Purple' & Unknown & Unknown & Unknown \\
\hline
\end{tabular}

Phytopathological status of Symphyotrichum spp. was monitored during the time period 2016-2018. Powdery mildew (caused by Erysiphe cichoracearum) was the most prominent and damaging the decorativeness of the plant (Fig. 1). S. dumosum, $S$. novi-belgii, S. ericoides, and $S$. tradescantii plants were the most seriously affected by the fungi (Table 3). Meanwhile, S. cordifolium, S. laeve, S. lanceolatum, S. lateriflorum, S. novae-angliae, S. oblongifolium, S. pilosum, S. $\times$ salignum, $S$. turbinellum, $S$. urophyllum did not show any symptoms of the disease.

Table 3. Susceptibility of different Symphyotrichum spp. to powdery mildew with respect to the growth period in the same site, (2016-2018).

\begin{tabular}{|l|c|c|c|}
\hline \multirow{2}{*}{ Species } & \multicolumn{3}{|c|}{ Susceptibility to powdery mildew, \% } \\
\cline { 2 - 4 } & 1st year & 2nd year & >3 year \\
\hline S. cordifolium & 0 & 0 & 00 \\
\hline S. dumosum & 5 & 15 & 10 \\
\hline S. ericoides & 0 & 0 & 0 \\
\hline S. laeve & 0 & 0 & 0 \\
\hline S. lanceolatum & 0 & 0 & 0 \\
\hline S. lateriflorum & 0 & 0 & 0 \\
\hline S. novae-angliae & 0 & 0 & 50 \\
\hline S. novi-belgii & 5 & 15 & 0 \\
\hline S. oblongifolium & 0 & 0 & 0 \\
\hline S. pilosum & 0 & 0 & 0 \\
\hline S. $\times$ salignum & 0 & 0 & 5 \\
\hline S. tradescantii & 0 & 0 & 0 \\
\hline S. turbinellum & 0 & 0 & 0 \\
\hline S. urophyllum & 0 & 0 & \\
\hline
\end{tabular}

Almost all plants planted in the first year were healthy and only some representatives of $S$. dumosum and $S$. novibelgii showed symptoms of powdery mildew injury. However, it should be acknowledged that the affected plants did not grow under ideal environmental conditions - in the shade, quite dry place, near to the trees. Powdery mildew became 
more prevalent in the following year of growth in the same site. The disease damaged the same species of Symphyotrichum: S. dumosum (15\%), and S. novi-belgii (15\%).

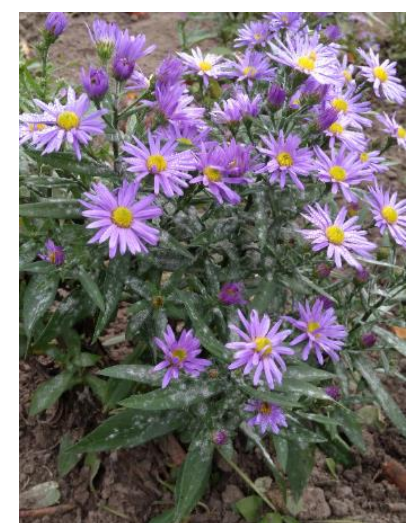

Figure 1. Leaves of Symphyotrichum sp. damaged by powdery mildew.

The third year of Symphyotrichum spp. growing in the same site was even more encouraging to the spread of powdery mildew. Up to 90 percent of $S$. dumosum plants were affected by the disease. Phytopathological status in $S$. novibelgii was slightly better: powdery mildew damaged a half of collection. However, symptoms of the disease were observed on the leaves of $S$. tradescantii $(5 \%)$ and $S$. ericoides $(10 \%)$. Thus, the longer period of growth in the same site, the more susceptible plants to the powdery mildew outbreak. S. cordifolium, S. laeve, S. lanceolatum, S. lateriflorum, S. novae-angliae, S. oblongifolium, S. pilosum, S. × salignum, S. turbinellum, S. urophyllum may be grown in the same site without replanting for more than three years.

\section{CONCLUSIONS}

1. Symphyotrichum spp. collection at Vytautas Magnus University Botanical Garden consists of 82 collection numbers: Symphyotrichum cordifolium, S. dumosum, S. ericoides, S. laeve, S. lanceolatum, S. lateriflorum, S. novae-angliae, S. novi-belgii, S. oblongifolium, S. pilosum, $S . \times$ salignum, S. tradescantii, S. turbinellum, S. urophyllum and their infraspecific taxa and cultones.

2. The collection has been accumulated through seed exchange with other botanical gardens and purchasing living plants from private collections, nurseries or other botanical gardens in Lithuania, Latvia, Estonia, Great Britain, Germany, Ukraine, Croatia, and Hungary.

3. S. cordifolium, S. laeve, S. lanceolatum, S. lateriflorum, S. novae-angliae, S. oblongifolium, S, pilosum, S. $\times$ salignum, $S$. turbinellum, and $S$. urophyllum demonstrated resistance to powdery mildew after growth in the same site for more than three years.

4. S. dumosum, S. novi-belgii, S. ericoides, and S. tradescantii showed susceptibility to powdery mildew during three years of growth without replantation.

\section{REFERENCES}

1. Cayuela L., Oksanen J. 2016. Taxonomic Standardization of Plant Species Names, Automated standardization of taxonomic names and removal of orthographic errors in plant species names using 'The Plant List' website (www.theplantlist.org), viewed 20 July 2019, http://www.theplantlist.org/browse/A/Compositae/Symphyotrichum/.

2. Hertlè B., Kyrmejeris,P., Nikig M. 1996. Sodo gèlès. UAB “Gamta”, 237 p. (In Lithuanian)

3. Kazlauskaitè, S. 2012. Želdiniu apsauga. Akademija. 193 p. (In Lithuanian)

4. Nesom G. L. 1994. Review of the taxonomy of Aster sensu lato (Asteraceae: Astereae), emphasizing the New World species, Phytologia, Vol. 77 (3), pp. 141-297.

5. Pirone P.P. 1978. Diseases and Pests of Ornamental Plants. Fifth Edition. New York, Chichester, Brisbane, Toronto, 566 p.

6. Vaidelys, J. 2005. Dekoratyviuju žoliniu augalu fenologiniu stebejimu, biometriniu matavimu ir sortimento sudarymo metodika. Kauno kolegijos leidybos centras, Mastaičiai, 83 p. (In Lithuanian) 